\title{
Leptoquark Production and Identification at High Energy Lepton Colliders $^{12}$
}

\author{
Michael A. Doncheski ${ }^{1}$ and Stephen Godfrey ${ }^{2}$ \\ ${ }^{1}$ Department of Physics, Pennsylvania State University, \\ Mont Alto, PA 17237 USA \\ ${ }^{2}$ Ottawa-Carleton Institute for Physics \\ Department of Physics, Carleton University, Ottawa CANADA, \\ $K 1 S 5 B 6$
}

\begin{abstract}
Leptoquarks can be produced in substantial numbers for masses very close to the collider centre of mass energy in $e^{+} e^{-}, e \gamma$, and $\mu^{+} \mu^{-}$collisions due to the quark content of the photon resulting in equivalently high discovery limits. Using polarization asymmetries in an $e \gamma$ collider the ten different types of leptoquarks listed by Buchmüller, Rückl and Wyler can be distinquished from one another for leptoquark masses essentially up to the kinematic limit. Thus, if a leptoquark were discovered, an $e \gamma$ collider could play a crucial role in determining its origins.
\end{abstract}

\section{INTRODUCTION}

There is considerable interest in the study of leptoquarks (LQs) which are colour (anti-)triplet, spin 0 or 1 particles that carry both baryon and lepton quantum numbers [1]. Such objects appear in a large number of extensions of the standard model such as grand unified theories, technicolour, and composite models. Quite generally, the signature for leptoquarks is very striking: a high $p_{T}$ lepton balanced by a jet (or missing $p_{T}$ balanced by a jet, for the $\nu q$ decay mode, if applicable). Present limits on leptoquarks have been obtained from direct searches at the HERA $e p$ collider, the Tevatron $p \bar{p}$ collider, and at the LEP $e^{+} e^{-}$collider [2] and estimates of future discovery limits for the LHC also exist [3]. In this paper we consider single leptoquark production in $\mu^{+} \mu^{-}, e^{+} e^{-}$, and $e \gamma$ collisions which utilizes the quark content of either a backscattered laser photon for the $e \gamma$ case or a WeizackerWilliams photon radiating off of one of the initial leptons for the $\mu^{+} \mu^{-}$or $e^{+} e^{-}$

1) Talk given at 20th Annual MRST Meeting on High-Energy Physics: MRST 98: Toward the Theory of Everything, Montreal, Canada, 13-15 May 1998

2) This research was supported in part by the Natural Sciences and Engineering Research Council of Canada. 
cases [4-6]. This process offers the advantage of a much higher kinematic limit than the LQ pair production process, is independent of the chirality of the LQ, and gives similar results for both scalar and vector leptoquarks.

Although the discovery of a leptoquark would be dramatic evidence for physics beyond the standard model it would lead to the question of which model the leptoquark originated from. Given the large number of leptoquark types it would be imperative to measure its properties to answer this question. There are 10 distinct leptoquark types which have been classified by Buchmüller, Rückl and Wyler

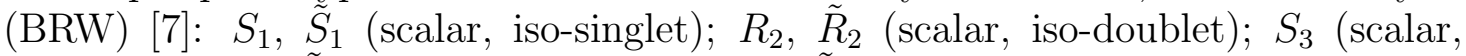
iso-triplet); $U_{1}, \tilde{U}_{1}$ (vector, iso-singlet); $V_{2}, \tilde{V}_{2}$ (vector, iso-doublet); $U_{3}$ (vector, iso-triplet). The production and corresponding decay signatures are quite similar, though not identical, and have been studied separately by many authors. The question arises as to how to differentiate between the different types. We show how a polarized $e \gamma$ collider can be used to differentiate the LQs. (ie. a polarized $e$ beam, like SLC, in conjunction with a polarized-laser backscattered photon beam.)

\section{LEPTOQUARK PRODUCTION}

The process we are considering is shown if Fig. 1. The parton level cross section for scalar leptoquark production is trivial, given by:

$$
\sigma(\hat{s})=\frac{\pi^{2} \kappa \alpha_{e} m}{M_{s}} \delta\left(M_{s}-\sqrt{\hat{s}}\right)
$$

where we have followed the convention adopted in the literature where the leptoquark couplings are replaced by a generic Yukawa coupling $g$ which is scaled to electromagnetic strength $g^{2} / 4 \pi=\kappa \alpha_{e m}$. We give results with $\kappa$ chosen to be $1 .{ }^{3}$ The cross section for vector leptoquark production is a factor of two larger. We only consider generation diagonal leptoquark couplings so that only leptoquarks which couple to electrons can be produced in $e \gamma\left(\right.$ or $e^{+} e^{-}$) collisions while for the $\mu^{+} \mu^{-}$ collider only leptoquarks which couple to muons can be produced. Convoluting the parton level cross section with the quark distribution in the photon one obtains the expression

$$
\sigma(s)=\int f_{q / \gamma}\left(z, M_{s}^{2}\right) \hat{\sigma}(\hat{s}) d z=f_{q / \gamma}\left(M_{s}^{2} / s, M_{s}^{2}\right) \frac{2 \pi^{2} \kappa \alpha_{e m}}{s} .
$$

The cross section depends on the LQ charge since the photon has a larger $u$ quark content than $d$ quark content.

For $e \gamma, e^{+} e^{-}$, and $\mu^{+} \mu^{-}$colliders the cross section is obtained by convoluting the expression for the resolved photon contribution to $e \gamma$ production of leptoquarks,

3) We note that the interaction Lagrangian used by Hewett and Pakvasa in Ref. [6] associates a factor $1 / \sqrt{2}$ with the leptoquark-lepton-quark coupling. 


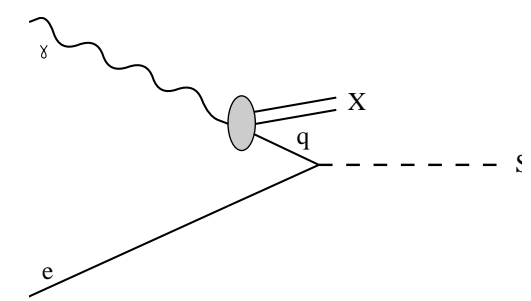

FIGURE 1. The resolved photon contribution for leptoquark production in $e \gamma$ collisions.

Eqn. (2), with, as appropriate, the backscattered laser photon distribution [8] or the Weizsäcker-Williams effective photon distribution: ${ }^{4}$

$$
\sigma\left(\ell^{+} \ell^{-} \rightarrow X S\right)=\frac{2 \pi^{2} \alpha_{e m} \kappa}{s} \int_{M_{s}^{2} / s}^{1} \frac{d x}{x} f_{\gamma / \ell}(x, \sqrt{s} / 2) f_{q / \gamma}\left(M_{s}^{2} /(x s), M_{s}^{2}\right) .
$$

Before proceeding to our results we consider possible backgrounds [9]. The leptoquark signal consists of a jet and electron with balanced transverse momentum and possibly activity from the hadronic remnant of the photon. The only serious background is a hard scattering of a quark inside the photon by the incident lepton via t-channel photon exchange; $e q \rightarrow e q$. By comparing the invariant mass distribution for this background to the LQ cross sections we found that it is typically smaller than the LQ signal by two orders of magnitude. Related to this process is the direct production of a quark pair via two photon fusion

$$
e+\gamma \rightarrow e+q+\bar{q}
$$

However, this process is dominated by the collinear divergence which is actually well described by the resolved photon process $e q \rightarrow e q$ given above. Once this contribution is subtracted away the remainder of the cross section is too small to be a concern [9]. Another possible background consists of $\tau$ 's pair produced via various mechanisms with one $\tau$ decaying leptonically and the other decaying hadronically. Because of the neutrinos in the final state it is expected that the electron and jet's $p_{T}$ do not in general balance which would distinguish these backgrounds from the signal. However, this background should be checked in a realistic detector Monte Carlo to be sure. The remaining backgrounds originate from heavy quark pair production with one quark decaying semileptonically and only the lepton being observed with the remaining heavy quark not being identified as such. All such backgrounds are significantly smaller than our signal in the kinematic region we are concerned with.

\section{LEPTOQUARK DISCOVERY LIMITS}

In Fig. 2 we show the cross sections for a $\sqrt{s}=1 \mathrm{TeV} e^{+} e^{-}$operating in both the backscattered laser $e \gamma$ mode and in the $e^{+} e^{-}$mode. The cross section for leptoquarks coupling to the $u$ quark is larger than those coupling to the $d$ quark. This

4) The effective photon distribution from muons is obtained by replacing $m_{e}$ with $m_{\mu}$. 

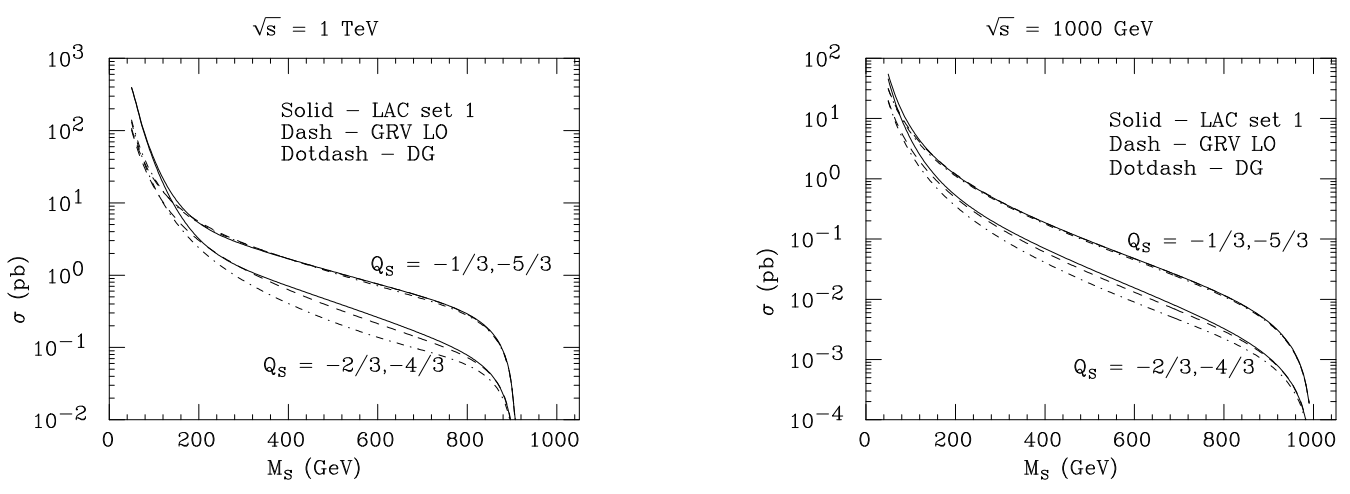

FIGURE 2. The cross sections for leptoquark production due to resolved photon contributions in $e \gamma$ collisions, for $\sqrt{s}_{e^{+} e^{-}}=1 \mathrm{TeV}$ with $\kappa$ chosen to be 1 . In the left figure the photon beam is due to laser backscattering and in the right figure it is given by the Weizsäcker-Williams effective photon distribution. In both cases the solid, dashed, dot-dashed line is for resolved photon distribution functions of Abramowicz, Charchula and Levy [14], Glück, Reya and Vogt [13], Drees and Grassie [12], respectively.

is due to the larger $u$ quark content of the photon compared to the $d$ quark content which can be traced to the larger $Q_{q}^{2}$ of the $u$-quark. There exist several different quark distribution functions in the literature [10-14]. For the four different leptoquark charges we show curves for three different distributions functions: Drees and Grassie (DG) [12], Glück, Reya and Vogt (GRV) [13], and Abramowicz, Charchula and Levy (LAC) set 1 [14]. The different distributions give almost identical results for the $Q_{L Q}=-1 / 3,-5 / 3$ leptoquarks and for the $Q_{L Q}=-2 / 3,-4 / 3$ leptoquarks give LQ cross sections that vary by most a factor of two, depending on the kinematic region. In the remainder of our results we will use the GRV distribution functions [13] which we take to be representative of the quark distributions in the photon.

Comparing the cross sections for the two collider modes we see that the kinematic limit for the $e \gamma$ mode is slightly lower than the $e^{+} e^{-}$mode. This is because the backscattered laser mode has an inherent energy limit beyond which the laser photons pair produce electrons. On the other hand the backscattered laser cross sections is larger than the $e^{+} e^{-}$mode. This simply reflects that the backscattered laser photon spectrum is harder than the Weizacker-Williams photon spectrum. To determine the leptoquark discovery limits for a given collider we multiply the cross section by the integrated luminosity and use the criteria that a certain number of signature events would constitute a leptoquark discovery. When we do this we find that the discovery limits for the $e \gamma$ and $e^{+} e^{-}$modes are not very different. Although the $e \gamma$ mode has a harder photon spectrum, the $e^{+} e^{-}$mode has a higher kinematic limit.

In Fig. 3 we plot the number of events for various collider energies for $e^{+} e^{-}, e \gamma$, and $\mu^{+} \mu^{-}$colliders. For the $\mu^{+} \mu^{-}$collider we used the $c$ and $s$ quark distributions 
TABLE 1. Leptoquark discovery limits for $e^{+} e^{-}, e \gamma$, and $\mu^{+} \mu^{-}$colliders. The discovery limits are based on the production of 100 LQ's for the energies and integrated luminosities given in columns one and two. The results were obtained using the GRV distribution functions [13].

\begin{tabular}{|c|c|c|c|c|c|}
\hline \multicolumn{6}{|c|}{$e^{+} e^{-}$Colliders } \\
\hline$\sqrt{s}(\mathrm{TeV})$ & $L\left(\mathrm{fb}^{-1}\right)$ & \multicolumn{2}{|c|}{ Scalar } & \multicolumn{2}{|c|}{ Vector } \\
\hline & & $-1 / 3,-5 / 3$ & $-4 / 3,-2 / 3$ & $-1 / 3,-5 / 3$ & $-4 / 3,-2 / 3$ \\
\hline$\overline{0.5}$ & 50 & 490 & 470 & 490 & 480 \\
\hline 1.0 & 200 & 980 & 940 & 980 & 970 \\
\hline 1.5 & 200 & 1440 & 1340 & 1470 & 1410 \\
\hline 5.0 & 1000 & 4700 & 4200 & 4800 & 4500 \\
\hline \multicolumn{6}{|c|}{ er Colliders } \\
\hline$\sqrt{s}(\mathrm{TeV})$ & $L\left(\mathrm{fb}^{-1}\right)$ & \multicolumn{2}{|c|}{ Scalar } & \multicolumn{2}{|c|}{ Vector } \\
\hline & & $-1 / 3,-5 / 3$ & $-4 / 3,-2 / 3$ & $-1 / 3,-5 / 3$ & $-4 / 3,-2 / 3$ \\
\hline$\overline{0.5}$ & 50 & 450 & 450 & 450 & 440 \\
\hline 1.0 & 200 & 900 & 900 & 910 & 910 \\
\hline 1.5 & 200 & 1360 & 1360 & 1360 & 1360 \\
\hline 5.0 & 1000 & 4500 & 4400 & 4500 & 4500 \\
\hline \multicolumn{6}{|c|}{$\overline{\mu^{+} \mu^{-} \text {Colliders }}$} \\
\hline$\sqrt{s}(\mathrm{TeV})$ & $L\left(\mathrm{fb}^{-1}\right)$ & \multicolumn{2}{|c|}{ Scalar } & \multicolumn{2}{|c|}{ Vector } \\
\hline & & $-1 / 3,-5 / 3$ & $-4 / 3,-2 / 3$ & $-1 / 3,-5 / 3$ & $-4 / 3,-2 / 3$ \\
\hline 0.5 & 0.7 & 250 & 170 & 310 & 220 \\
\hline 0.5 & 50 & 400 & 310 & 440 & 360 \\
\hline 4.0 & 1000 & 3600 & 3000 & 3700 & 3400 \\
\hline
\end{tabular}

in the photon rather than the $u$ and $d$ quark distributions since we only consider generation diagonal leptoquark couplings. For $\sqrt{s}=500 \mathrm{GeV}$ a $e^{+} e^{-}$collider will have about a $25 \%$ higher reach than a $\mu^{+} \mu^{-}$collider due to the larger $u$ and $d$ distributions arising from the smaller quark masses. For the highest energy lepton colliders considered the differences become relatively small. For the high luminosities being envisaged, the limiting factor in producing enough leptoquarks to meet our discovery criteria is the kinematic limit. Because, for a given $e^{+} e^{-}$ centre of mass energy, an $e^{+} e^{-}$collider will have a higher energy than an $e \gamma$ collider using a backscattered laser, the $e^{+} e^{-}$collider will have a higher discovery limit. Finally, note that the discovery limit for vector leptoquarks is slightly higher than the discovery limit for scalar leptoquarks. This simply reflects the fact that the cross section for vector leptoquarks is a factor of two larger than the cross section for scalar leptoquarks. We summarize the discovery limits for the various colliders in Table 1. The OPAL [17] and DELPHI [18] collaborations at LEP have obtained leptoquark limits using the process we have described. 

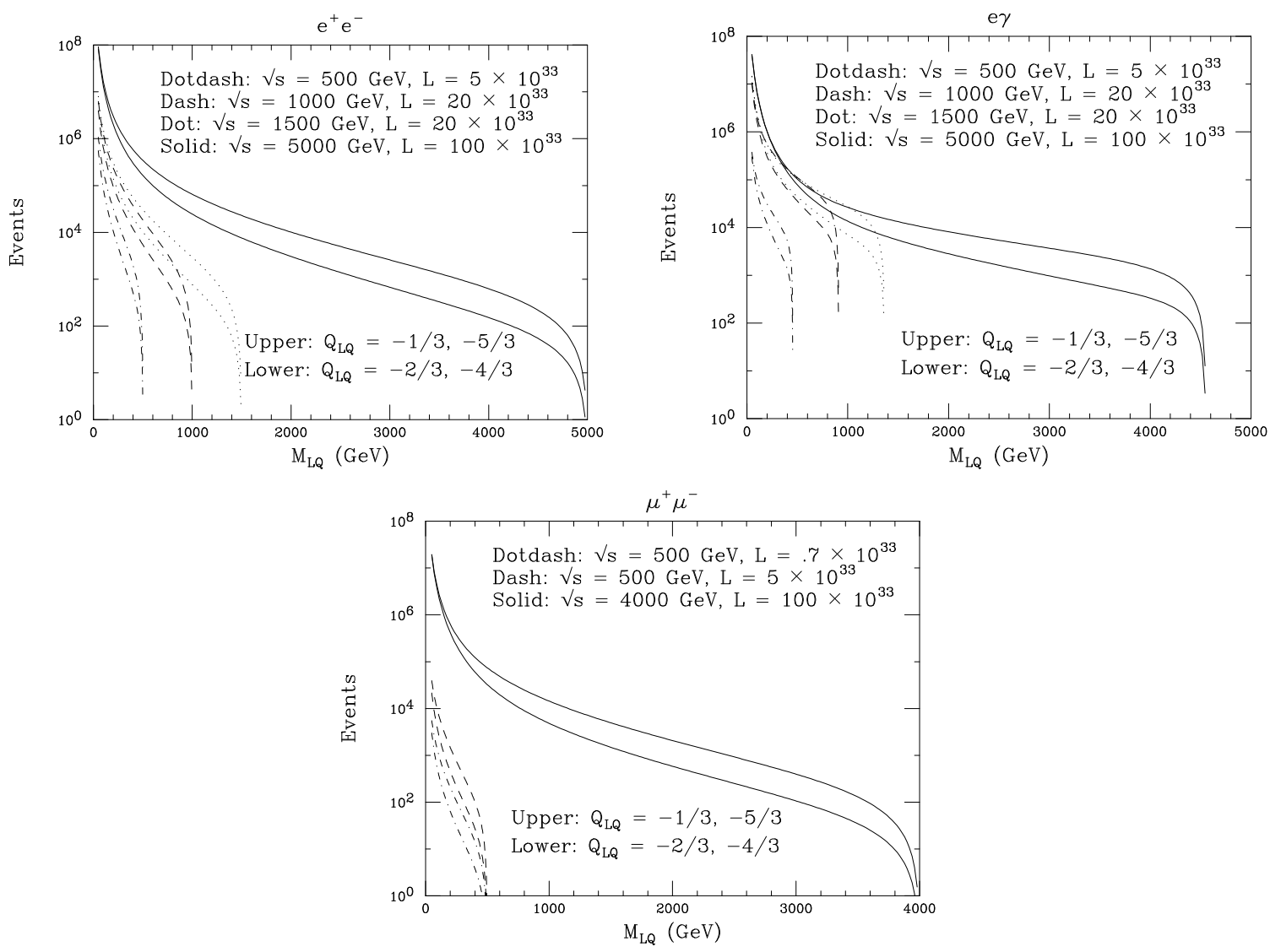

FIGURE 3. Event rates for single leptoquark production in $e^{+} e^{-}, e \gamma$, and $\mu^{+} \mu^{-}$collisions. The centre of mass energies and integrated luminosities are given by the line labelling in the figures. The results were obtained using the GRV distribution functions [13]. Note that only 2nd generation LQ's would be produced in $\mu^{+} \mu^{-}$collisions with our assumption of generation diagonality.

\section{LEPTOQUARK IDENTIFICATION}

If a leptoquark were actually discovered the next step would be to determine its properties so that we could determine which model it originated from. We will assume that a peak in the $e+$ jet invariant mass is observed in some collider (i.e., the existance of a LQ has been established), and so we need simply to identify the particular type of LQ. We assume that the leptoquark charge has not been determined and assume no intergenerational couplings. Furthermore, we will assume that only one of the ten possible types of LQs is present. Table 2 of BRW [7] gives information on the couplings to various quark and lepton combinations; the missing (and necessary) bit of information in BRW is that the quark and lepton have the same helicity (RR or LL) for scalar LQ production while they have opposite helicity (RL or LR) for vector LQ production. It is then possible to construct the cross sections for the various helicity combinations and consequently the double 
spin asymmetry [5], for the different types of LQs.

Thus, a first step in identifying leptoquarks would be to determine the coupling chirality, ie. whether it couples to $e_{L}, e_{R}$, or $e_{U}$. This could be accomplished by using electron polarization either directly or by using a left-right asymmetry measurement:

$$
A^{+-}=\frac{\sigma^{+}-\sigma^{-}}{\sigma^{+}+\sigma^{-}}=\frac{C_{L}^{2}-C_{R}^{2}}{C_{L}^{2}+C_{R}^{2}}
$$

This divides the 10 BRW leptoquark classifications into three groups:

$e_{L}^{-}: \quad \tilde{R}_{2}, S_{3}, U_{3}, \tilde{V}_{2}$

$e_{R}^{-}: \quad \tilde{S}_{1}, \tilde{U}_{1}$

$e_{U}^{-}: U_{1}, V_{2}, R_{2}, S_{1}$

We can further distinguish whether the leptoquarks are scalar or vector. This could be accomplished in two ways. In the first one can study the angular distributions of the leptoquark decay products. In the second we can use the double asymmetry:

$$
A_{L L}=\frac{\left(\sigma^{++}+\sigma^{--}\right)-\left(\sigma^{+-}+\sigma^{-+}\right)}{\left(\sigma^{++}+\sigma^{--}\right)+\left(\sigma^{+-}+\sigma^{-+}\right)}
$$

where the first index refers to the electron helicity and the second to the quark helicity. Because scalars only have a non-zero cross section for $\sigma^{++}$and $\sigma^{--}$for scalar LQ's the parton level asymmetry for $e q$ collisions is $\hat{a}_{L L}=+1$. Similarly, since vectors only have a non-zero cross section for $\sigma^{+-}$and $\sigma^{-+}$for vector LQ's $\hat{a}_{L L}=-1$.

To obtain observable asymmetries one must convolute the parton level cross sections with polarized distribution functions. Doing so will reduce the asymmetries from their parton level values of \pm 1 so one must determine whether the observable asymmetries can distinguish between the leptoquark types. The expressions for the double longitudinal spin asymmetry $A_{L L}$ are given in Ref. [5]. In Figure 4 we plot $A_{L L}$ for the $e \gamma$ collider which started with $\sqrt{s}_{e^{+} e^{-}}=1 \mathrm{TeV}$. To obtain these curves we used parameterizations of the asymptotic polarized photon distribution functions $[15,16]$, where it is assumed that $Q^{2}$ and $x$ are large enough that the Vector Meson Dominance part of the photon structure is not important, but rather the behavior is dominated by the point-like $\gamma q \bar{q}$ coupling. In order to be consistent, we used a similar asymptotic parameterization for the unpolarized photon distribution functions as well [10], even though various sets of more correct photon distribution functions exist (e.g., [11-14]). We only used this asymptotic approximation in the unpolarized case for the calculation of the asymmetry, where it is hoped that in taking a ratio of the asymptotic polarized to the asymptotic unpolarized photon distribution functions, the error introduced will be minimized. Still, we suggest that our results be considered cautiously, at least in the relatively small LQ mass region. We note that in the asymptotic approximation, the unpolarized photon distribution functions have (not unexpectedly) a similar form to the polarized photon distribution functions. 

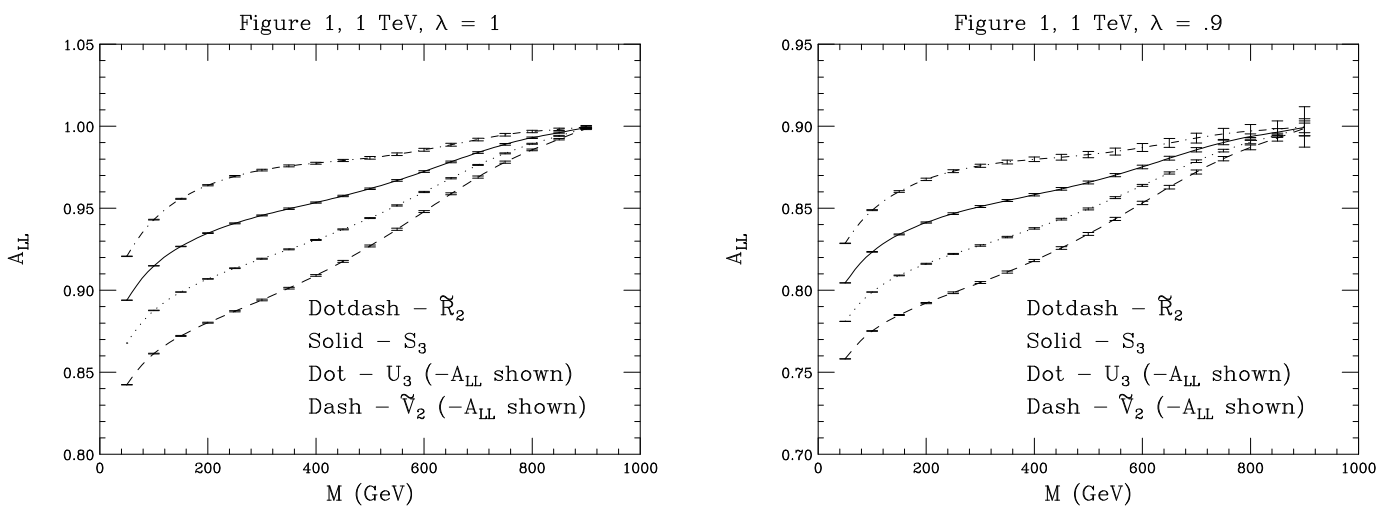

FIGURE 4. $A_{L L}$ vs $M_{L Q}$ for a $1 \mathrm{TeV}$ e $\gamma$ collider. The statistical errors are based on $200 \mathrm{fb}^{-1}$. The left figure is for $100 \%$ polarization and the right for $90 \%$ polarization.

In Fig. 4 we show asymmetries for 100\% polarization and for $90 \%$ polarization which is considered to be achievable given the SLC experience. The error bars are based on an total integrated luminosity of $200 \mathrm{fb}^{-1}$ for $\sqrt{s}_{e^{+} e^{-}}=1 \mathrm{TeV}$. In these figures note that we are showing $-A_{L L}$ for the vector cases so that we can use a larger scale. Quite clearly, polarization would enable us to distinguish between vector and scalar. For the cases where there are two types of leptoquarks of the same chiral couplings, for example the scalar isodoublet $\tilde{R}_{2}$ and scalar isotriplet $S_{3}$, we could distinguish between them up to about $3 / 4$ the kinematic limit.

Finally, one additional bit of information to further differentiate among the various possible leptoquarks is to search for the $\nu q^{\prime}$ decay mode. This signature is quite similar to a supersymmetric particle decay (high $p_{T}$ jet plus missing $p_{T}$ ) so that although it cannot be used to unambiguously determine the existence on leptoquarks, it can be used, in conjunction with the observation of an approximately equal number of $e q$ events (as expected in some models) to provide further information on leptoquark couplings. Taken together, the leptoquark type can be uniquely determined. If more than one leptoquark were discovered, determining their properties would tell us their origin and therefore, the underlying theory.

\section{SUMMARY}

To summarize, we have presented results for single leptoquark production in $e \gamma$, $e^{+} e^{-}$, and $\mu^{+} \mu^{-}$collisions. The discovery limits for leptoquarks is very close to the centre of mass energy of the colliding particles. It also appears that a polarized e $\gamma$ collider can be used to differentiate between the different models of LQs that can exist, essentially up to the kinematic limit. Furthermore, it is quite easy to distinguish scalar LQs from vector LQs for all LQ mass (given that the LQ is kinematically allowed). Thus $e^{+} e^{-}, e \gamma$, and $\mu^{+} \mu^{-}$colliders have much to offer in the searches for leptoquarks. If leptoquarks were discovered, $e \gamma$ colliders could play a crucial role in unravelling their properties, and therefore the underlying physics. 


\section{REFERENCES}

1. See, for example, J.L. Hewett and T.G. Rizzo, hep-ph/9708419 and references therein.

2. Particle Data Group, C. Caso et al, The European Physical Journal C3, 1 (1998).

3. See for example the ATLAS Technical Proposal, W.W. Armstrong et al., CERN Report CERN/LHCC/94-43; J.L. Hewett, T.G. Rizzo, S. Pakvasa, H.E. Haber and A. Pomarol, Proceedings of the Workshop on Physics at Current Accelerators and Supercolliders, ed. J.L. Hewett, A.R. White, and D. Zeppenfeld, Argonne report ANL-HEP-CP-93-92, p. 539.

4. M. A. Doncheski and S. Godfrey, Phys. Rev. D49, 6220 (1994) [hep-ph/9311288]; Phys. Lett. B393, 355(1997), [hep-ph/9608368]; Mod. Phys. Lett. A12, 1859(1997), [hep-ph/9704380].

5. M. A. Doncheski and S. Godfrey, Phys. Rev. D51, 1040 (1995) [hep-ph/9407317].

6. O.J. Éboli, E.M. Gregores, M.B. Magro, P.G. Mercadante, and S.F. Novaes, Phys. Lett. B311, 147 (1993); H. Nadeau and D. London, Phys. Rev. D47, 3742 (1993); G. Bélanger, D. London and H. Nadeau, Phys. Rev. D49, 3140 (1994); J.L. Hewett and S. Pakvasa, Phys. Lett. B227, 178 (1989); F. Cuypers, [hep-ph/9602355]. Other related papers are: J. E. Cieza Montalvo and O.J.P. Éboli, Phys. Rev. D47, 837 (1993); T.M. Aliev and Kh.A. Mustafaev, Yad. Fiz. 58, 771 (1991); V. Ilyin et al., Phys. Lett. B351, 504 (1995); erratum B352, 500 (1995); Phys. Lett. B356, 531 (1995).

7. W. Buchmüller, R. Rückl, and D. Wyler, Phys. Lett. B191, 442 (1987).

8. I.F. Ginzburg et al., Nucl. Instrum. Methods, 205, 47 (1983); 219, 5 (1984); C. Akerlof, Ann Arbor report UM HE 81-59 (1981; unpublished).

9. Hadronic backgrounds in $e^{+} e^{-}$and $e \gamma$ collisions and associated references are given in M.A. Doncheski, S. Godfrey, and K.A. Peterson, Phys.Rev. D55, 183 (1997) [hep-ph/9407348].

10. A. Nicolaidis, Nucl. Phys. B163, 156 (1980).

11. D.W. Duke and J.F. Owens, Phys. Rev. D26, 1600 (1982).

12. M. Drees and K. Grassie, Z. Phys. C28, 451 (1985); M. Drees and R. Godbole, Nucl. Phys. B339, 355 (1990).

13. M. Glück, E. Reya and A. Vogt, Phys. Lett. B222, 149 (1989); Phys. Rev. D45, 3986 (1992); Phys. Rev. D46, 1973 (1992).

14. H. Abramowicz, K. Charchula, and A. Levy, Phys. Lett. B269, 458 (1991).

15. J.A. Hassan and D.J. Pilling, Nucl. Phys. B187, 563 (1981).

16. Z. Xu, Phys. Rev. D30, 1440 (1984).

17. OPAL Collaboration, OPAL Physics Note 288 (1997).

18. DELPHI Collaboration, DELPHI 97-112 Conf 94 (1997). 